\title{
Distribución y Hábitat de las algas de las costas de Melilla
}

\author{
Angel Granda Vera
}

\section{Introducción}

Este estudio, realizado hace un par de años y que no ha podido ver la luz por diferentes motivos, pretende suministrar datos sobre un grupo de seres vivos, quizás no muy conocidos; al mismo tiempo servir como un punto de referencia, para un mejor conocimiento de la distribución de las especies de algas, a ambos lados del estrecho; también como una contribución para despertar el interés por el entorno natural de Melilla, para una mejor conservación del mismo.

Los medios han sido muy precarios, lo que ha dificultado la recogida de datos, sobre todo a partir de cierta profundidad; es evidente que la investigación se refiere a la línea de costa que corresponde a Melilla, con alguna aportación con el fin de contrastar, de la distribución de las diferentes especies, al otro lado del cabo Tres Forcas; este hecho permitió comprobar, la mayor riqueza del lado oeste de dicho cabo, así como una abundante presencia de géneros atlánticos como Fucus ( $F$. spiralis), o Culteria multifida que no he detectado en el lado este.

\section{Situación y costas de Melilla}

La ciudad está situada en el norte de Africa, sobre la base este del cabo Tres For. cas, encima de una pequeña península, de unos treinta metros de altura a $2^{\circ} 56^{\prime} 24^{\prime \prime}$ de longitud este y $35^{\circ} 17^{\prime} 40^{\prime \prime}$ norte. Parte de la costa estudiada es artificial y coincide en general con las zonas más degradadas; el resto, la misma que luego continúa en territorio marroquí, está formada por calizas miocénicas y areniscas amarillentas que han sufrido un fuerte levantamiento geológico.

Las aguas cálidas, normalmente limpias, están afectadas por corrientes de origen atlántico (lo que explica la presencia de especies procedentes de ese océano), así como otras del Mediterráneo septentrional y oriental, aunque su intensidad no sea muy fuerte, si condicionan, junto a la disposición del cabo Tres Forcas, el depósito continuo de sedimentos, que han cerrado la Mar Chica, al generar una barra litoral.

\section{Zonas de muestreo y distribución por especies}

Se eligieron cinco puntos característicos y representativos, atendiendo a criterios 
tales como claridad de las aguas, limpieza, grado de contaminación, zonas a mar abierto o de ensenada, etc.; también se recogieron ejemplares de arribazón. En cada uno de los lugares se procuró recoger información de cada uno de los niveles que se pueden distinguir en una costa: franja supralitoral, zona medio litoral y franja infralitoral.

Otro dato significativo ha sido buscar la posible relación entre especies, para ver si la presencia de una cualquiera, condicionaba la presencia de otra de alguna forma.

La distribución observada por especie es la siguiente:

Reino Protistas. Algas:

División Clorofitos; Clase Cloroficeas

1) Ulva lactuca (Linnè): presente en casi todos los lugares estudiados; en la mayor parte de los casos aislada, $y$ en ocasiones asociada a otras algas como Coralina officinalis, o Scytosiphon lomentaria.

2) Enteromorpha linza (Linnè): normalmente se encuentra en charcas y en zonas del medio litoral superior; generalmente en masas y a plena luz.

3) Chaetomorpha linum (O.F. Muller): repartida a lo largo de toda la costa, formando tapices sobre las rocas del medio litoral superior.

4) Enteromorpha intestinalis (Linnè): en aguas poco profundas y en oquedades cerca del borde marino, que son inundadas intermitentemente, por el oleaje o las mareas; a veces en aguas algo contaminadas.

5) Valonia utricularis (Roth): no muy abundante, y en lugares muy concretos; hábitat en la zona infralitoral y en lugares de intensa corriente y poca luz.

6) Cladophora prolifera. Se han localizado ejemplares aislados en puntos de fuerte rompiente y con abundante luminosidad.

7) Dasycladus clavaeformis (Roth): se encuentran grupos aislados en la zona infralitoral; poseen fuertes estipites.

8) Acetabularia mediterrónea (Lamouroux): una de las especies más abundantes, formando pequenas "praderas" en sitios de aguas tranquilas, o en grupos mezcladas con otras especies; condiciones de luminosidad no muy estrictas, estando presente en las zonas infra y medio litorales.

9) Derbesia marina (Lyngbie) Solier: presencia abundante; recubre rocas en lugares de calma, que parte del día no están directamente en contacto con el agua, en la zona medio litoral superior.

10) Codium tomentosum Stackhouse: presente de forma aislada o en pequeños grupos en hendiduras rocosas, o sobre rocas diseminadas en fondos arenosos; en general poco exigentes, en cuanto a luminosidad.

11) Codium bursa (Linne) C. A. Agardh. Sobre rocas en la zona infralitoral; en bastantes ocasiones encontrada de arribazón en las playas; en general en sitios iluminados.

12) Codium difforme. Fijada en zonas de oleaje del litoral medio, formando cojinetes y asociada a otras algas. 


\section{División Feofios; clase Feoficeas}

13) Scytosiphon lomentaria (Lyngbie) Link.: encontrada cubriendo grandes extendiones, asociada a otras especies, en la zona medio litoral superior.

14) Arthorocladia villosa (Hudson) Duby: crece formando "praderas" en la zona infralitoral, generalmente combinada con Halopteris filicina; poco exigente en cuanto al factor luz.

15) Desmarestia ligulata (Lightfoot) Lamouroux.: poco abundante, se encuentra en lugares con poca luminosidad del medio litoral, asociada a veces a Coraltima officinalis y a Pterocladia capillacea.

16) Sargassum vulgare (J.G. Agardh): la mayor parte de las ocasiones hallada de arribazón; en contadas veces fija sobre rocas a una cierta profundidad.

17) Sargassum hornischuchi (C.A. Agardh): de distribución parecida a la anterior, pero más localizadas en la zona medio litoral, en rocas expuestas al oleaje y con luminosidad; en algunos casos asociada a Cystoseira fimbriata.

18) Halopteris filicina (Grattan). Kutzing.: ampliamente distribuida con un hábitat preferente en la zona infralitoral, con luminosidad media; en ocasiones aparece junto a Arthrocladia villosa.

19) Cladostephus verticillatus (Lightfoot) C.A. Agardh.: presente en la zona infralitoral, en sitios poco luminosos.

20) Dictyopteris membranacea (Stackhouse) Batters.: especie con fuertes estípites que le permite soportar la batida del oleaje sobre las rocas en que se fija; generalmente en lugares luminosos del litoral medio superior.

21) Dictyota dichotoma (Hudson) Lamouroux.: muy abundante pero sin formar espesos grupos; habitát fundamental en las zonas medio litoral inferior e infralitoral, con luminosidad media; a veces asociada a Corallina officinalis.

22) Padina pavonia (Linné) Lamouroux.: presencia generalizada en todos los biotopos, incluso en los más contaminados de la zona infralitoral; en ocasiones formando pequeñas "praderas" junto a Acetabularia mediterranea.

23) Cystoseira tamariscifolia (Hudson) Papenfuss (=C. ericoides): especie rara hallada en la zona medio litoral y normalmente de arribazón.

24) Cystoseira fimbriata (Desfontaine) Bory (=C. abrotanifolia).: es la especie más abundante de este género; se encuentra en la zona medio litoral superior, con luminosidad y a veces junto a Sargassum hornschuchi.

\section{Division Rodofitos; clase Rodoficeas}

25) Pterocladia capillacea (Gmelin) Bornet \& Thuret (P. pinnata).: se halla en sitios de calma del litoral medio superior, expuestos a poca luz; a menudo aparece junto a Corallina officinalis y a Desmarestia ligulata.

26) Plocamium coccineum(Hudson) Lyngbie (=P. cartilagineum, P. vulgare) se encuentra en lugares oscuros sobre rocas en el infralitoral solitariamente en general.

27) Corallina officinalis (Linné): especie abundante, tanto en la zona litoral superior como en la infralitoral; forma masas calcáreas sobre las que se asientan otras algas.

28) Peysonnelia squamaria (Gmelin) Decaisne.: normalmente asentada sobre rocas de la zona infralitoral, y a veces en la arena bajo ellas. 
29) Jania rubens (Linné) Lamouroux (=Corallina rubens): en masas sobre rocas en la zona infralitoral con poca luminosidad.

30) Pseudolithophyllum expansum: forma costras calcáreas de tono rosáceo, sobre las rocas.

31) Ceramium rubrum (Hudson) C.A. Agardh.: especie que se encuentra generalmente aislada, y ocasionalmente asociada a Corallina officinalis; en las zonas mediolitoral e infralitoral.

32) Gelidium latifolium (Greville) Bornet \& Thuret.: localizada muy raramente, de forma aislada en la zona infra litoral.

Breve comentario sobre la distribución en cada uno de los puntos estudiados

\section{Punto 1: Dique sur.}

Zona artificial formada por rocas basálticas, que constituye un parapeto contra la entrada de arena y la erosión de las aguas; actualmente favorece el aumento de la contaminación y un escaso movimiento de corrientes, debido a la construcción de un puerto, paralelo a él, en el vecino Marruecos.

En este punto son abundantes las Clorofíceas y las Rodofíceas; donde la luz es más tenue se encuentran Corallina officinalis y $C$. rubrum; alli donde la luminosidad es más fuerte, están presentes $C$. officinalis, Ulva lactuca y Codium difforme; la variedad de especies es más pobre que en otros puntos, quizás debido al mayor grado de contaminación del lugar.

\section{Punto 2: Escollera del puerto y faro.}

Los materiales que los forman son conglomerados de cementos y gravas, con fondos escalonados de bloques de basalto. La luminosidad es bastante alta en este dique nordeste, con aguas más limpias que le baten continuamente; hallamos aquí numerosas especies que poseen fuertes estípites, o forman cojinetes sobre las rocas; la cobertura y la diversidad son altas (siempre relativamente) y la contaminación escasa o nula.

Las especies dominantes son: en la zona infralitoral, Halopteris filicina, Dyctiota dichotoma y Padina pavonia y en la medio litoral, $C$. difforme y $C$. officinalis; las principales asociaciones se dan entre esta última especie y $C$. difforme y $U$. lactuca.

\section{Punto 3: Boca del León y Trápana.}

Zona natural constituída por calizas miocénicas, con fondos arenosos y aporte de materiales con efectos contaminantes; numerosas especies con medios de sujeción para soportar el continuo embate del mar. $C$. officinalis es la especie más abundante en el medio litoral, y $H$. filicina en la infralitoral; la primera en muchos casos junto a Derbesia marina y $U$. lactuca donde hay luminosidad apreciable, y junto a $C$. rubrum en lugares más oscuros.

La cobertura es alta, aunque hay sitios con el sustrato móvil, por lo que no están colonizados por las algas; la diversidad no es muy alta. 
Punto 4: Ensenada de los Galápagos.

La bahía presenta alto índice de contaminación, debido al aporte de colectores; ello afecta a las algas al igual que a otras muchas especies de seres vivos, de manera negativa; por otra parte hay un fuerte depósito de sedimentos (principalmente arenas), lo que unido a la contaminación ya apuntada anteriormente, hacen que las aguas sean turbias y la luminosidad escasa.

Las especies con mayor presencia son: $C$. difforme y Acetabularia mediterranea en la zona infralitoral, y $C$. officinalis y $C$. difforme en la medio litoral; en la entrada de la ensenada la riqueza de especies es superior, dominando $C$. difforme, $A$. mediterranea y H. filicina.

\section{Punto 5: Aguadú.}

Se pueden distinguir dos partes según la constitución de los fondos: una zona izquierda formada por calizas miocénicas con una disposición en planchas que permiten extensas "praderas" de algas sobre ellas; la zona derecha constituida por areniscas, con presencia de detritus procedentes del vertedero de basuras y zona de tiro militar. La primera con una gran riqueza de especies, mientras que en la segunda hay poca variedad; el sustrato en ambas es mixto, con partes rocosas y fondos de arena.

U. lactuca, C. oficinalis Derbesia marina es la asociación más frecuente en la zona izquierda, y las dos primeras especies en la zona derecha.

En cuanto a dominancia, en la zona medio litoral hay una fuerte presencia de $U$. lactuca, $C$. officinalis, y en la infralitoral de Halopteris filicina y Cladostephum verticillatum, así como extensiones de A. mediterranea y Padina pavonia. 
Símbolos empleados en los cortes esquemáticos:

Acetabularia mediterránea

Arthrocladia villosa ...

Chaetomorpha linum

Ceramium rubrum

Cladophora prolífera

Cladostephus verticillatus

Cystoseira fimbriata

Cystoseira tamariscifolia

Codium bursa

Codium difforme

Codium tomentosum

Corallina officinalis

Dasycladus clavaeformis

Derbesia marina

Desmarestia ligulata

Dictyopteris membranacea

Dyctiota dichótoma

Enteromorpha linza

Enteromorpha intestinalis

Halopteris filicina

Jania rubens

Padina pavonia

Peysonnelia squamaria

Plocamium coccineum

Pseudolithophilum expansum

Pterocladia capillácea

Sargassum hornschunchi

Sargassum vulgare

Scytosiphon lomentaria

Ulva lactuca

Valonia utricularis 


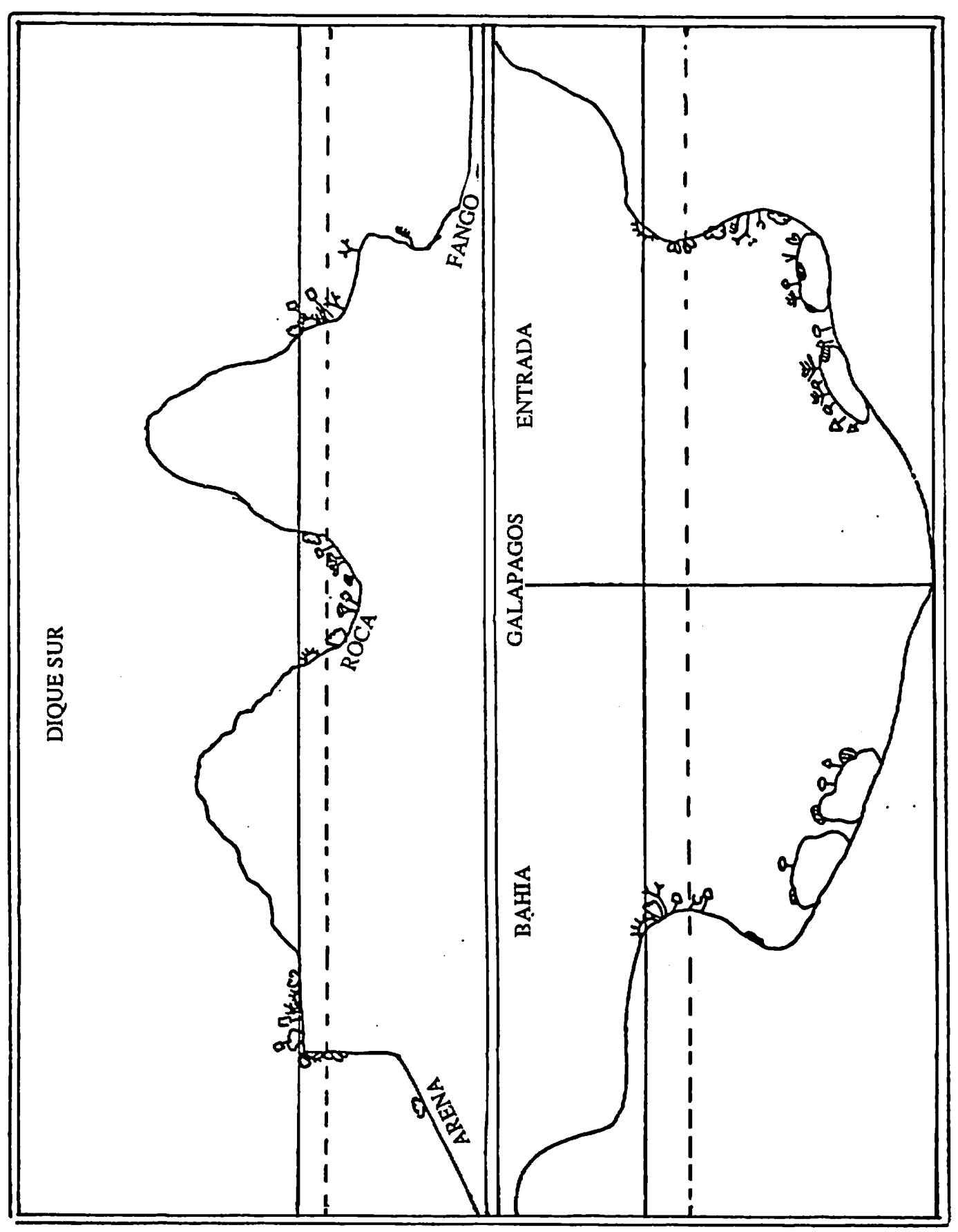




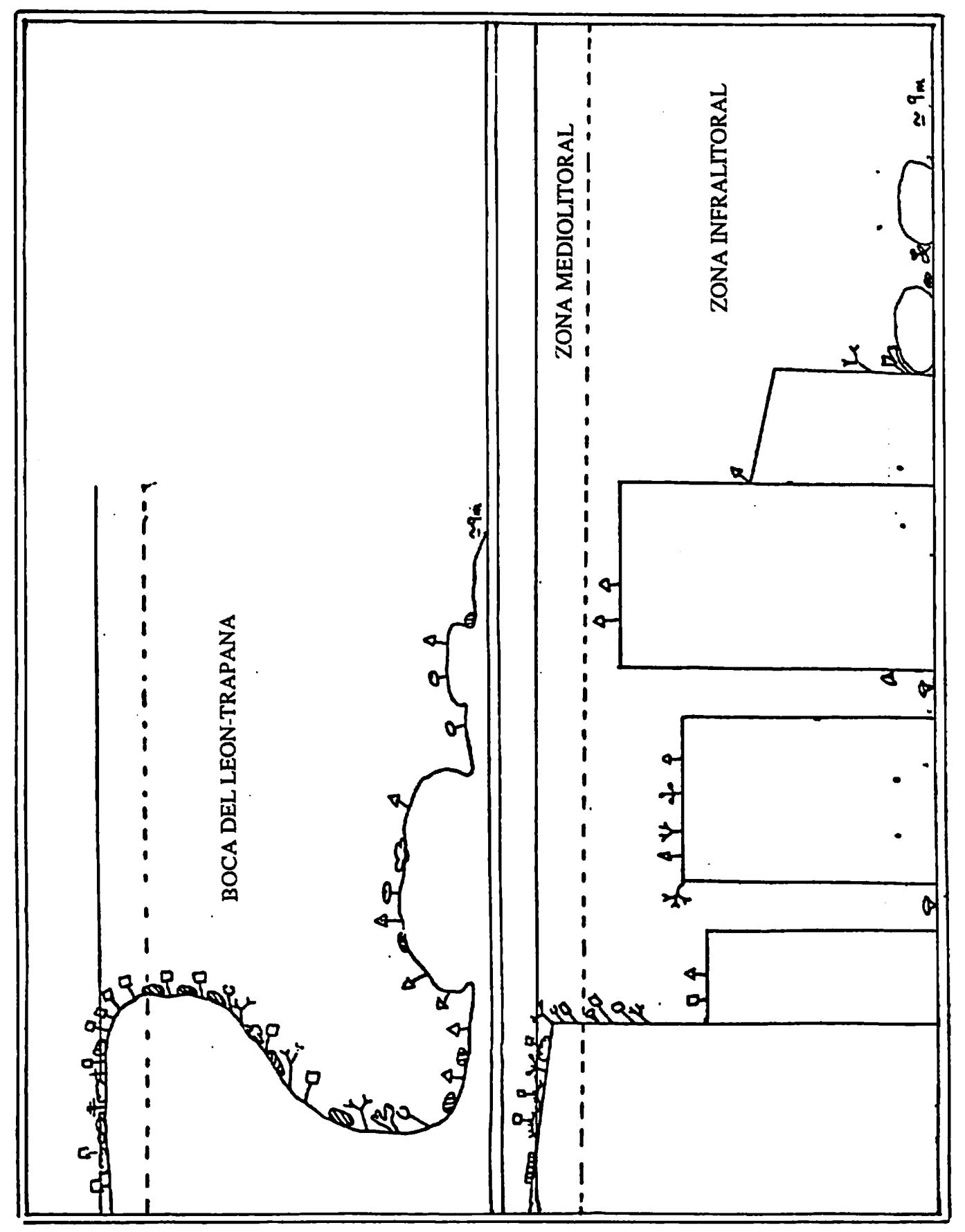




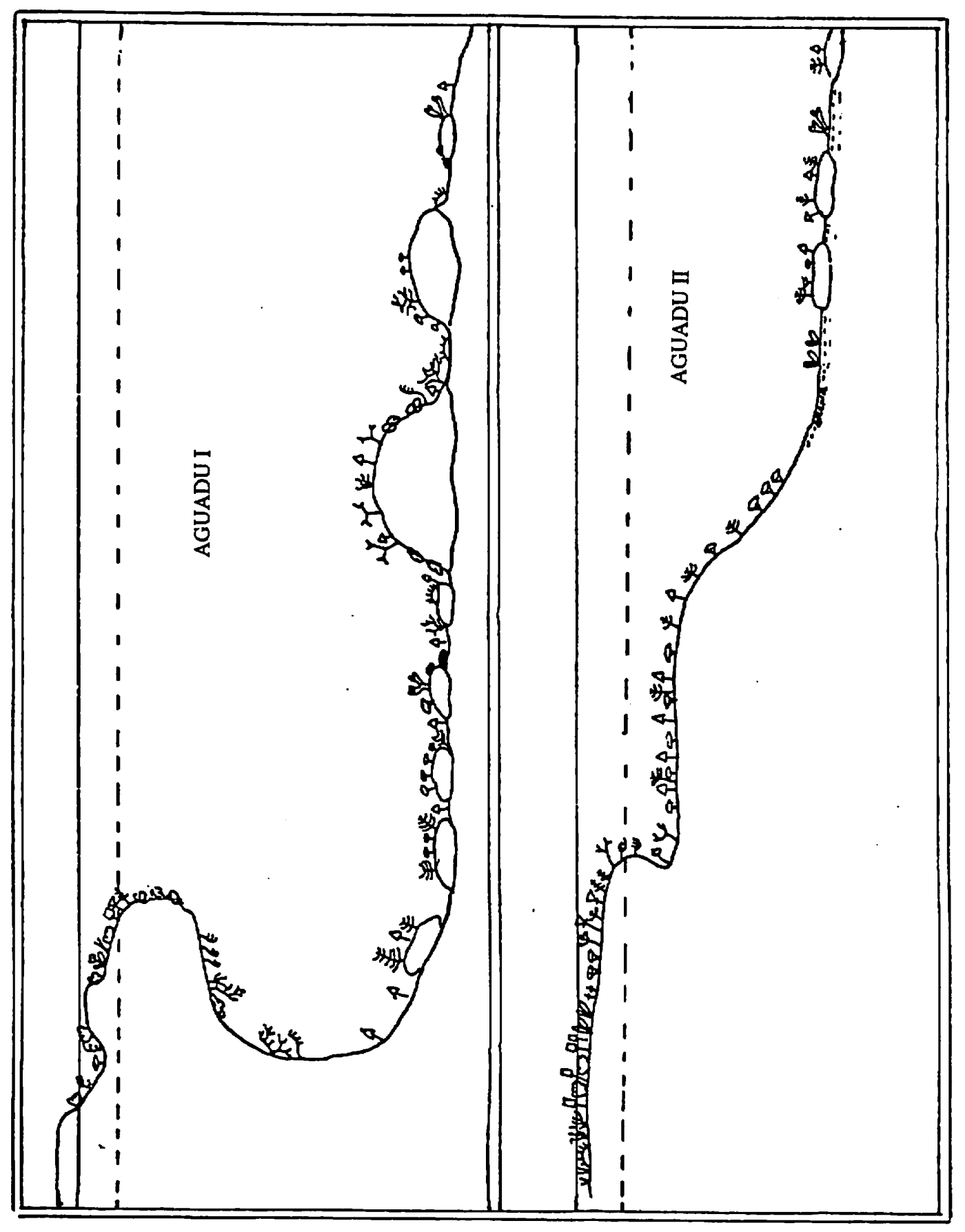




\section{Conclusiones}

La presencia de bastantes especies en nuestras costas, incluso de especies atlánticas en ocasiones, es importante a mi juicio; las distribuciones siguen en general una gradación condicionada por la luminosidad, siendo las Clorofíceas predominantes en las zonas litoral y medio litoral, las Feoficeas en esta última y en la infralitoral, y las poblaciones de Rodoficeas colonizando sustratos más profundos; existen excepciones como la presencia de $C$. officinalis en todos los niveles, incluso en superficie o que especies de Feofíceas y Rodofíceas ocupen biotopos menos profundos, cuando las condiciones de luminosidad, son semejantes a las que soportan a mayor profundidad.

En la región infralitoral la dominancia de especies como Arthrocladia villosa y $H$. filicina es común a toda la costa, junto a Jania rubens y Plocamium coccineum, además de acompañantes casuales.

Es también importante señalar, como la movilidad y dureza y consistencia del sustrato favorece la determinada existencia de especies con poderosos estípites, impidiendo que otras poblaciones específicas más beneficiadas en aguas tranquilas, se desarrollen; tal parece ser el caso de $C$. officinalis, alga calcárea con estípite incrustante.

Por último sefralar el grado de contaminación como factor ecológico negativo, en la presenciá y abundancia de los distintos grupos de algas; la dominancia en exceso de Cloroficeas (relativamente), incluso en niveles en los que se encuentran normalmente, ligado a la disminución de las poblaciones de Feofíceas (indicativo de zonas marinas contaminadas), permiten asegurar que en nuestras costas hay lugares degradados ecológicamente; en este sentido el ejemplo de la Ensenada de los Galápagos es el más claro. 